\title{
Análise do tempo de reação a partir do desempenho motor de adolescentes praticantes do nado Crawl
}

\author{
Analysis of reaction time performance engine from the practitioners of \\ teenagers swim crawl
}

\author{
Guilherme Ribas Smidt ${ }^{1}$, Ellen dos Santos Soares ${ }^{1 *}$, Ana Carla Piovesan ${ }^{1}$, Ana Cristina Al- \\ pes $^{1}$, Marta Rodrigues da Silva ${ }^{1}$, Sara Teresinha Corazza ${ }^{1}$
}

ARTIGO ORIGINAL | ORIGINAL ARTICLE

\begin{abstract}
O Este estudo teve como objetivo avaliar o tempo de reação simples (TRS) e de escolha (TRE) e o desempenho no nado crawl em diferentes níveis de aprendizagem. Fizeram parte deste estudo 26 sujeitos com idade média de 12.15 anos divididos em dois grupos: iniciantes (G1) e avançados (G2). Avaliou-se o tempo de reação (TR) por meio de um software e o nado crawl com o Teste de Desempenho Motor do Nado Crawl. Os resultados demonstraram diferença no TRS com média de G1 $=.341 \mathrm{~s}$ e G2 $=.271 \mathrm{~s}(t$ $=-3.670 ; p=.001 ; \mathrm{TE}=0.60)$ e no TRE com média de $\mathrm{G} 1=.651 \mathrm{~s}$ e $\mathrm{G} 2=.492 \mathrm{~s}(t=-4.016 ; p=.001$; $\mathrm{TE}=0.63)$, indicando que no estágio avançado os aprendizes possuem melhores tempos de resposta. Uma correlação moderada encontrou-se entre G1 (iniciante) e TRE (avançado) $(r=-0.560 ; p=0.046)$, e G2 (avançado) e TRS (avançado) $(r=-0.685 ; p=0.010)$. Conclui-se que o TR é importante para o sucesso na performance do nado crawl uma vez que seu aprimoramento resulta em menores gastos de tempo em indivíduos em estágio avançado.

Palavras-chave: Tempo de reação, Destreza Motora, Natação, Aprendizagem.
\end{abstract}

ABSTRACT

The aim of this study was to evaluate the simple reaction time (SRT), the choice reaction time (CRT) and the performance of front crawl swimming at different learning levels. This study had 26 participants of both genders with a mean age of 12.15 years, divided in 2 groups: beginners (G1) and advanced (G2). Reaction time (RT) was assessed by a software that measures the interval between a visual stimulus and response, and the performance of front crawl swimming was measured by a test which allows to evaluate and score the swimming technique. The Student $t$ test for independent samples was used and significance level was set at $5 \%$. Results showed that there was a difference between the 2 groups in SRT (G1 $=.341 \mathrm{~s}$ e $\mathrm{G} 2=.271 \mathrm{~s} ; t=-3.070 ; p=.001 ; \mathrm{TE}=0.60)$, and CRT Time $(\mathrm{G} 1=.651 \mathrm{~s}$ e G2 $=.492 \mathrm{~s} ; t=-$ 4.016; $p=.001 ; \mathrm{TE}=0.63$ ). A moderate correlation was found between $\mathrm{G} 1$ (beginner) and TRE (Advanced) $(r=-0.560, p=0.046)$, and G2 (advanced) and TRS (Advanced) $(r=-0.685, p=0.010)$. We conclude that TR is important for success in the front crawl performance since its improvement results in lower time costs in subjects at an advanced stage.

Keywords: Reaction Time, Motor Dexterity, Swimming, Learning.

\footnotetext{
Artigo recebido a 03.07.2013; Aceite a 24.09.2014

${ }^{1}$ Centro de Educação Física e Desporto. Universidade Federal de Santa Maria (UFSM), Rio Grande do Sul, Brasil.

* Autor correspondente: Rua Luiz Castanha, 180. Bairro Km3. Santa Maria, Rio Grande do Sul, Brasil. E-mail: ellensoa@gmail.com
} 


\section{INTRODUÇÃO}

Desde a sua concepção o indivíduo passa por mudanças que constantemente alteram a interação com o ambiente e com as tarefas físicas e mecânicas. As mudanças no repertório motor, próprias da aprendizagem, ocorrem no número, na complexidade e na qualidade de execução das ações motoras (Canfield, 2000; Wulf, Shea, \& Lewthwaite, 2010). Mudanças de comportamento são analisadas considerando aqueles movimentos mais complexos dentro da estrutura do movimento, denominadas habilidades motoras. Estas podem ser definidas como atividades ou execuções que têm uma meta definida a ser atingida, como, nadar, dirigir, jogar futebol, entre outras (Dayan \& Cohen, 2011; Magill, 2000). Além disso, as habilidades motoras também estão relacionadas às características que distinguem o nível de proficiência demonstrada por um executante (Bôscolo, Santos, \& Oliveira, 2011; Schmidt \& Wrisberg, 2001).

Neste contexto, de acordo com a aprendizagem das habilidades motoras, certas características do movimento são modificadas, o que acontece, normalmente, em uma sequência hierárquica (Pellegrini, 2000; Pereira, Teixeira, \& Corazza, 2012). Pellegrini (2000) classifica as etapas pelas quais passam os aprendizes em três estágios, definidos como iniciante, intermediário e avançado, cada um com características definidas. No primeiro estágio, o executante parece descoordenado, com movimentos desnecessários e sem fluência, não se detém a detalhes, têm dificuldade de identificar os estímulos importantes para a ação e apresenta grande quantidade de erros. Já no segundo estágio o aprendiz vai eliminando os movimentos desnecessários, procurando focar-se nos estímulos e nos detalhes importantes, diminuindo os erros apresentados. Desta forma, a sequência de movimentos ganha fluência e harmonia, que conjuntamente com a progressiva estabilização do padrão motor, resulta na poupança de tempo e energia. Por fim, no último estágio, o aprendiz já tem certeza de como se executa a habilidade, o padrão motor é estável e, por isso, utiliza um mínimo de energia para uma execução eficiente (Pellegrini, 2000).
É importante realçar que a aquisição e o aperfeiçoamento das habilidades motoras dependerão da base motora de cada indivíduo, juntamente com o desenvolvimento das capacidades motoras que são essenciais, uma vez que uma habilidade motora pode ter, na sua estrutura, o envolvimento de várias capacidades (Pereira et al., 2012). As capacidades motoras são qualidades inatas de uma pessoa relacionadas ao seu desempenho na execução de tarefas e, por isso, aparecem subjacentes ao sucesso na aprendizagem e desempenho motor (Dayan \& Cohen, 2011; Magill, 2000; Wulf et al., 2010).

No que se refere a natação, uma das capacidades motoras necessárias para seu desenvolvimento é o tempo de reação (TR), preponderante na fase de partida (Miyamoto \& Meira, 2004; Pereira, Teixeira, Villis, \& Corazza, 2009). A mensuração do TR fornece um esclarecimento dos processos internos que ocorrem no movimento voluntário, determinando a velocidade e a eficácia da tomada de decisão (Martins, Dascal, Bruzi, Caldeira, \& Turetta, 2010; Morales, Maciel, Silva, \& Silva, 2011).

Como uma capacidade perceptivo-motora, o tempo de reação representa o intervalo de tempo entre a apresentação de um estímulo e o início do movimento, que é a resposta gerada. Pode ser dividido em tempo de reação simples (TRS), quando se utiliza somente um estímulo ou sinal e somente uma resposta; tempo de reação de escolha (TRE), quando são apresentados dois ou mais estímulos e duas ou mais respostas são possíveis, sendo necessário optar pela mais adequada; ou ainda, de descriminação, quando há mais de um sinal, mas somente uma resposta possível (Magill, 2000; Morales et al., 2011; Schmidt \& Wrisberg, 2001). Sobre este especto, tendo em conta que as capacidades motoras, como o TR, se podem modificar nos diferentes estágios de aprendizagem, procuramos com este estudo avaliar o tempo de reação a partir do desempenho motor de praticantes do nado crawl em diferentes estágios de aprendizagem.

\section{METODOLOGIA}

Trata-se de um estudo descritivo de caráter qualitativo (Thomas, Nelson, \& Silverman, 
2007), que procurou analisar o tempo de reação e descrever as características do desempenho motor do nado crawl de praticantes de natação.

\section{Participantes}

Os participantes foram selecionados de maneira intencional pelo critério de voluntariado em três academias da cidade de Santa Maria - RS. O grupo foi composto por 26 praticantes de natação, 13 do sexo feminino (11.69 \pm 1.03 anos de idade), e 13 do sexo masculino (12.07 \pm 1.25 anos de idade). Como critérios de inclusão adotou-se: faixa etária entre 11 e 14 anos; estar envolvido com a prática de natação há no mínimo seis meses e no máximo dois anos; não fazer uso de nenhum medicamento de uso contínuo; não apresentar patologias crônicas e/ou osteo-musculares que impossibilitassem a realização dos testes.

Todos os indivíduos participavam das atividades de natação com frequência de duas vezes semanais, no turno contrário ao horário escolar e relataram não participar de outras atividades extracurriculares. No que se refere à prática da natação, todas as instituições selecionadas seguiam um programa similar para o desenvolvimento das aulas em relação às atividades e exercícios propostos.

\section{Procedimentos}

O estudo obedeceu às normas e critérios exigidos pela lei 196/96 do Conselho Nacional de Saúde - CNS. O projeto de pesquisa foi submetido ao Comitê de Ética em Pesquisas em Seres Humanos da Universidade Federal de Santa Maria - UFSM, e aprovado sob o protocolo $\mathrm{n}^{\circ}$ 003.0.243.000-0. Todos os participantes assinaram um Termo de Consentimento Livre e Esclarecido (TCLE).

A coleta dos dados foi realizada, individualmente, nas dependências das academias. Inicialmente os sujeitos foram avaliados quanto ao tempo de reação, em uma sala reservada em um ambiente calmo e tranquilo, onde não houve interferência de estímulos externos e ambientais; e, em seguida, foram realizadas as avaliações do desempenho do nado crawl nas piscinas.

A partir da avaliação das características do nado crawl, foram formados dois grupos com 13 indivíduos cada: G1 (iniciante), com idade média de 11.61 anos e tempo de prática 6 meses; e G2 (avançado), com idade média de 12.69 anos e tempo de prática 2 anos. Embora a diferença na faixa etária seja pequena entre os grupos, as características das atividades desenvolvidas na natação são diferenciadas de acordo com o nível dos aprendizes e o seu tempo de prática. No grupo iniciante, eram realizadas aulas com material didático de apoio (prancha), exercitando membros inferiores e membros superiores de forma isolada, treinando a respiração na borda da piscina, concomitantemente com o ensino do nado crawl. Já no grupo avançado, as atividades eram voltadas principalmente para o aperfeiçoamento da técnica do nado, sempre junto ao aprimoramento dos fundamentos.

\section{Instrumentos}

Para avaliação do nado crawl e classificação dos estágios de aprendizagem, utilizou-se um teste criado e validado por Corazza, Pereira, Villis, e Katzer (2006). O teste é composto por seis itens subdivididos em: (1) posição do corpo; (2) movimentos das pernas; (3) fase não propulsiva dos braços; (4) fase propulsiva dos braços (tração e empurre); (5) respiração e sincronização de braços e pernas; (6) respiração. Cada item do teste é subdividido em outros subitens, que no total podem pontuar o desempenho do nado de 0 a 29 pontos. Os indivíduos foram avaliados durante a execução do nado, por um avaliador treinado, no qual foi verificada para cada subitem do teste a execução correta ou não conforme a sua descrição. Em caso de execução correta era atribuído um ponto, caso contrário era atribuído o valor zero.

A partir da pontuação obtida, os indivíduos são classificados quanto aos estágios de aprendizagem em iniciante, intermediário ou avançado. No estágio iniciante, são classificados os desempenhos de 0 a 9 pontos; no intermediário aqueles com pontuação entre 10 e 19 pontos; e no avançado os que obtiveram pontuação entre 20 e 29. Além da pontuação mínima, para ser classificado como avançado, o indivíduo não pode obter zero em nenhum subitem do teste.

A avaliação do Tempo de Reação Simples e do Tempo de Reação de Escolha foi realizado 
por meio de um software específico criado e validado por Pereira, Dias, e Corazza (2007), desenvolvido na ferramenta Borland Delphi 7, que utiliza a linguagem de programação object pascal. O software avalia o tempo de reação a partir de um estímulo visual que deve ser respondido, após surgir na tela, o mais rápido possível com a utilização dos botões do rato do computador. A resposta é a reação do movimento do membro superior dominante, calculando assim o tempo entre a apresentação do estímulo e o início do movimento ou resposta.

\section{Análise Estatística}

Primeiramente por meio do teste de Shapiro Wilk, foi verificada a normalidade dos dados. Assumidos os pressupostos de normalidade ( $p$ $>$.05) foi aplicado o teste $t$ para amostras independentes com o objetivo de verificar a diferença entre os grupos. Para análise da correlação utilizou-se o coeficiente de correlação de Pearson. Foi utilizado um programa estatístico SPSS, versão 14.0, com nível de significância de $5 \%$.

Tabela 1

Valores descritivos referentes à pontuação no teste de desempenho motor do nado crawl.

\begin{tabular}{lcccc}
\hline Item do teste & \multicolumn{2}{c}{ (G1) Iniciante } & \multicolumn{2}{c}{ (G2) Avançado } \\
& Min-máx & Média & Min-máx & Média \\
\hline 1- Posição do corpo & $0-2$ & 1.15 & $2-3$ & 2.84 \\
2- Movimento das pernas & $1-2$ & 1.07 & $2-4$ & 3.69 \\
3- Fase não propulsiva dos braços & $1-3$ & 1.69 & $3-6$ & 4.92 \\
4- Fase propulsiva dos braços & $1-3$ & 1.53 & $3-8$ & 5.84 \\
5- Respiração & $0-3$ & 1.53 & $2-3$ & 2.92 \\
6- Sincronização & $0-1$ & .76 & $3-6$ & 5.00 \\
\hline Pontuação Total & $3-9$ & 7.92 & $21-30$ & 25.38 \\
\hline
\end{tabular}

No que se refere a análise do tempo de reação os valores são apresentados nas tabelas 2 e 3. Considerando que para o tempo de reação, quanto menor o tempo, melhor o desempenho, evidenciou-se por meio do teste t de student diferenças estatísticas significativas tanto no TRS $(t=-3.670 ; p=.001)$, quanto no TRE $(t=-$ $4.016 ; p=.001)$ em benefício do grupo avançado. Foram obtidos valores elevados para a

\section{RESULTADOS}

Para o desenvolvimento do estudo, inicialmente, todos os sujeitos foram avaliados quanto ao desempenho motor do nado crawl, de modo que os resultados obtidos no teste fossem utilizados para a classificação quanto ao estágio de aprendizagem e posterior divisão nos grupos iniciante (G1) e avançado (G2). Nesse sentido, quanto ao desempenho motor do nado crawl, conforme é possível observar na tabela 1, a média em pontos que gerou o G1 foi visivelmente inferior a média que gerou o G2 em todos os sub-itens do teste, o que resultou em uma pontuação média total de 7.92 pontos e 25.38 pontos para cada grupo, respetivamente. A partir destes valores obtidos foi feita a classificação dos grupos, na qual a baixa pontuação alcançada pelo G1 caracterizou o estágio iniciante em que os aprendizes se encontram, do mesmo modo que as pontuações mais altas do G2 caracterizam o estágio avançado da aprendizagem. magnitude do efeito tanto para o TRS (0.60), como para o TRE (0.63).

Através da análise correlacional constatou-se moderada correlação entre o desempenho no teste de nado crawl do G2 (avançado) e TRS (avançado) ( $\mathrm{r}=-0.685 ; \mathrm{p}=0.010)$, considerando que no estágio avançado o tempo de reação foi menor. 
Tabela 2

Tempo de reação simples (TRS) e do Tempo de reação de escolha (TRE) dos grupos iniciante e avançado

\begin{tabular}{llllllllcc}
\hline & Grupo & $\mathrm{N}$ & Mínimo & Máximo & Média & Desvio Padrão & $T E$ & $t$ & $p$ \\
\hline \multirow{2}{*}{ TRS } & $\mathrm{G} 1$ & 13 & $.226 \mathrm{~s}$ & $.430 \mathrm{~s}$ & $.341 \mathrm{~s}$ & .062 & .60 & $-3.670^{*}$ & .001 \\
& $\mathrm{G} 2$ & 13 & $.223 \mathrm{~s}$ & $.324 \mathrm{~s}$ & $.271 \mathrm{~s}$ & .029 & & & \\
\hline
\end{tabular}

G1: Iniciante; G2: Avançado; $\mathrm{s}=$ segundos; *Diferença significativa para $p<.05$ (teste $t$ de Student para amostras independentes), TE $=$ Tamanho do efeito.

Tabela 3

Tempo de reação de escolha (TRE) dos grupos iniciante e avançado

\begin{tabular}{lllllllllc}
\hline & Grupo & $\mathrm{N}$ & Mínimo & Máximo & Média & Desvio Padrão & $T E$ & $t$ & $p$ \\
\hline TRE & G1 & 13 & $.441 \mathrm{~s}$ & $.822 \mathrm{~s}$ & $.651 \mathrm{~s}$ & .105 & .63 & $-4.016^{*}$ & .001 \\
& $\mathrm{G} 2$ & 13 & $.327 \mathrm{~s}$ & $.683 \mathrm{~s}$ & $.492 \mathrm{~s}$ & .096 & & & \\
\hline
\end{tabular}

G1: Iniciante; G2: Avançado; $\mathrm{s}=$ segundos; ${ }^{*}$ Diferença significativa para $p<.05$ (teste $t$ de Student para amostras independentes), $\mathrm{TE}=$ Tamanho do efeito.

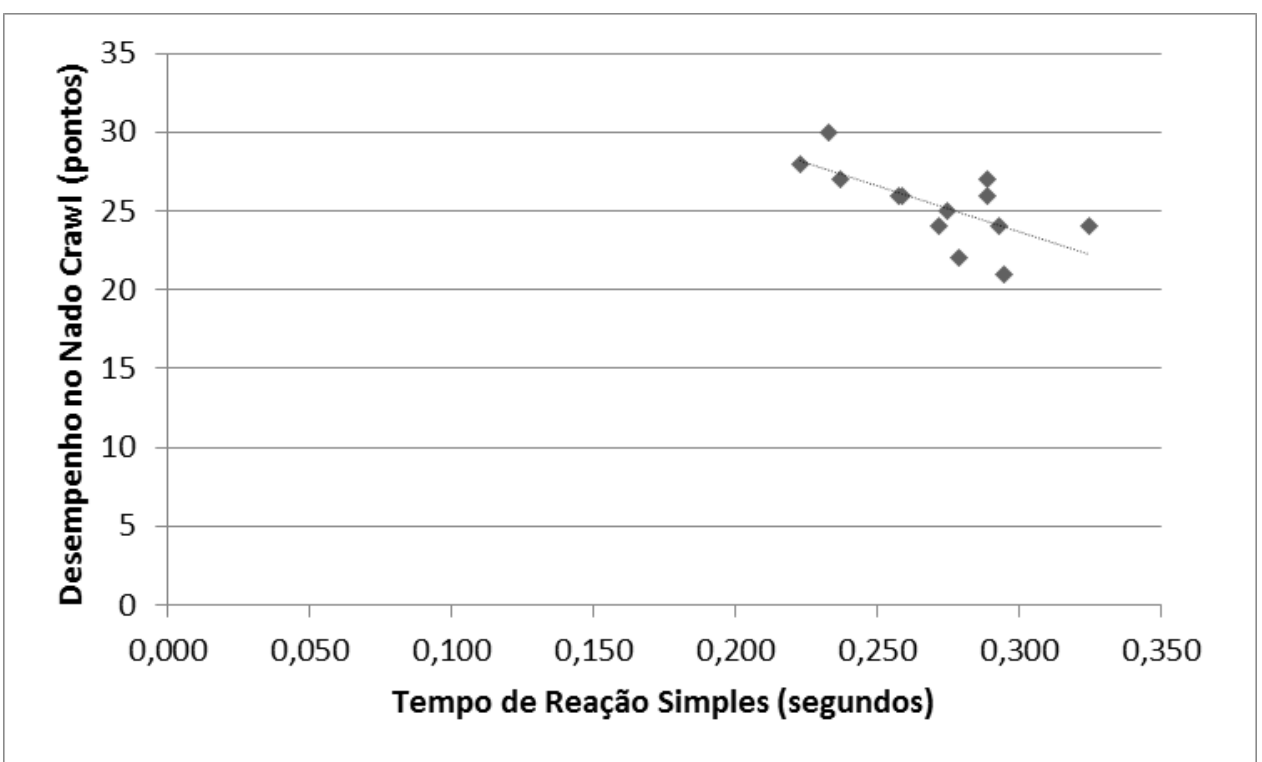

Figura 1. Correlação de Pearson entre Tempo de Reação Simples e Desempenho no nado Crawl.

\section{DISCUSSÃo}

O tempo de reação motora é uma medida bastante utilizada em pesquisas como um indicador do desempenho sensório-motor. Investigações em torno desta temática são importantes para a compreensão de como o sistema nervoso identifica informações relevantes presentes no meio ambiente e seleciona as ações motoras mais adequadas para a resposta (Araujo \& Carreiro, 2009; Hedvall et al., 2013; Penke et al., 2010). Nesse sentido, o presente estudo buscou identificar este processo de perceção e resposta motora em indivíduos em diferentes níveis de aprendizagem, levando em consideração a bagagem motora adquirida por meio da prática.

As análises iniciais foram para a classificação dos grupos em relação aos estágios de aprendizagem. O G1 obteve uma média 7.92 pontos, sendo classificado como iniciante e o G2 teve média de 25.38 pontos, sendo classificado como avançado de acordo com as características do movimento do nado Crawl avaliadas. O teste de desempenho motor do nado crawl foi utilizado em estudos anteriores (Corazza et al., 2006; Pereira et al., 2007) também com o objetivo de identificar e classificar indivíduos quanto ao nível de aprendizagem.

Para Freudenheim et al. (2005), não é necessário utilizar equipamentos sofisticados para distinguir indivíduos em diferentes estágios de aprendizagem, uma vez que apresentam características claramente observáveis. Neste mesmo aspeto, Bôscolo, Santos, e Oliveira (2011) chamam a atenção para a necessidade de professores e pesquisadores buscarem identificar as características do executante em cada um dos diferentes estágios da aprendizagem, visto que este é um dos fatores que permitem determinar 
corretamente a progressão de atividades no ensino de habilidades motoras aquáticas.

Na habilidade de nadar, a padronização espaço-temporal dos movimentos caracteriza de forma evidente. O processo de aquisição dessa habilidade pode ser descrito como cíclico e dinâmico de estabilidade-instabilidade-estabilidade, direcionado a um maior grau de complexidade. Sendo assim, um indivíduo com um padrão avançado de nado Crawl passou por mais ciclos de estabilidade e quebra de estabilidade que um iniciante, na mesma habilidade (Freudenheim et al., 2005; Seifert, Boulesteix, Chollet, \& Vilas-Boas, 2008; Seifert, Leblanc, Chollet, \& Delignières, 2010).

Em relação às análises de tempo de reação, os resultados encontrados revelaram que, quanto melhor o desempenho no nado crawl, melhor o tempo de reação simples e de escolha, uma vez que os valores foram significativamente menores $(p=.001)$ no grupo avançado em relação ao grupo iniciante. Nesse caso, uma vez que o TR representa o tempo necessário para a execução de uma resposta, quanto menor for o tempo de reação maior será a eficiência dos mecanismos e processos centrais (Deary, Liewald, \& Nissan, 2011; Droit-Volet \& Zélanti, 2013; Kaufman, DeYoung, Gray, Brown, \& Mackintosh, 2009; Takeuchi et al., 2011).

As diferenças estatisticamente significativas encontradas entre os grupos podem estar relacionadas a uma possível programação motora incompleta no início da aprendizagem (Pereira et al., 2009), o que acarreta dificuldades em identificar os estímulos internos ou externos que são mais relevantes para a ação o qual leva a um dispêndio de tempo excessivo (Pellegrini, 2000). Estes resultados vão ao encontro de outros estudos desenvolvidos com enfoques parecidos (Mori, Ohtani, \& Imanaka, 2002; Pereira et al., 2009; Vaghetti, Roesler, \& Andrade, 2007). Vaghetti, Roesler, e Andrade (2007) realizou uma análise de tempo de reação simples auditivo e visual em surfistas profissionais, amadores e praticantes e foram encontradas diferenças estatisticamente significativas para $o$ TRS auditivo e visual entre profissionais do sexo masculino versus praticantes e entre profissionais do sexo feminino versus praticantes.
Entre os amadores versus praticantes verificou diferenças significativas apenas para TRS visual com TRS menores para os mais experientes, ou seja, os indivíduos com mais habilidade na prática do surf.

Outro estudo foi realizado por Pereira, Teixeira, Villis, e Corazza (2009) com o objetivo de investigar a relação entre os resultados dos tempos de reação simples e de escolha com o desempenho motor do nado crawl em jovens em diferentes estágios de aprendizagem. Igualmente os melhores resultados foram significativos tanto no TRS $(\mathrm{r}=-0.192 \mathrm{p}=0.284)$ quanto no TRE $(r=-0.471, p=0.006)$ para o grupo no estágio avançado. Neste mesmo contexto, embora não tenham analisado atletas de natação, Mori, Ohtani, e Imanaka (2002), encontraram diferenças estatísticas significativas quando avaliaram atletas de karatê, sendo que os menores tempos foram dos mais experientes.

O TR pode ser utilizado como medida de eficiência dos processos de antecipação e tomada de decisão. Dessa forma, estímulos desconhecidos necessitam de um maior TR em comparação a estímulos conhecidos (Macedo, Covre, Orsati, Oliveira, \& Schwartzman, 2007). No presente estudo constatou-se moderada correlação entre o desempenho no nado crawl e o TRS no grupo avançado $(r=-0.685 ; p=0.010)$, considerando-se que quanto maior o estágio para uma habilidade, menor será o tempo de reação. Estudo de Lidor, Argov, e Daniel (1998) salienta que a quantidade de prática representa um aspeto que pode interferir no TR, pois a prática auxilia o indivíduo a discernir os aspetos relevantes de um estímulo diminuindo o número de incertezas no estágio de seleção de respostas, reduzindo o intervalo de tempo no estágio de programação do movimento, aumentando a eficiência da performance e a diminuição do TR.

Um estudo de Miyamoto e Meira (2004), realizado com atletas federados e não-federados, reforçou que para atletas federados foram detetadas correlações significativas e positivas, porém fracas, entre tempo de reação de membros inferiores (TRMI) e tempo de movimento nos 100 metros (TM100) e entre tempo de movimento de 50 metros (TM50) e (TM100). No que tange ao grupo de não federados, foi identificada 
apenas uma correlação significativa e negativa, porém fraca, entre tempo de reação de membros superiores (TRMS) e (TM100), é relatado que embora os atletas federados deste estudo apresentem melhor tempo de movimento (TM) em relação aos não federados, o tempo de reação (TR) não se configura como uma variável determinante de diferenças de desempenho entre velocistas de níveis diferentes. Contudo no presente estudo a variável TR esteve fortemente associada ao nível de estágio do executante. Dessa forma, podemos inferir que o principal fator de influência sobre o TR são os mecanismos centrais de processamento antecedentes ao movimento.

Vaghetti et al. (2007) encontraram uma correlação positiva para o TRS visual entre os profissionais (femininos) versus no ranking $(\mathrm{r}=$ $0.269 \mathrm{p}=0.204)$. Os processos fisiológicos de transformação dos estímulos auditivos e visuais em resposta motora são idênticos, em todas as modalidades desportivas; o diferencial está no produto final, que é a mecânica do movimento, ou seja, os grupamentos musculares que são recrutados para realizar determinadas tarefas. Os TRS estão associados à automatização dos gestos desportivos. Este estudo não corrobora com o presente estudo, o qual encontrou correlações negativas.

De maneira geral, no presente estudo realizado com adolescentes, fica evidenciado que mudanças ocorrem nas capacidades motoras, como o tempo de reação, em decorrência da prática, o que reforça a importância do treinamento. A adolescência é definida pela Organização Mundial da Saúde (OMS) como o período compreendido entre 10 e 19 anos de idade. Sabe-se que nessa etapa, o crescimento e o desenvolvimento biológico são fortemente influenciados pela interação de fatores genéticos e ambientais (Fortes, Miranda, Amaral, \& Ferreira, 2011), de modo que o nível de maturação somado às exigências específicas do treinamento de cada grupo poderão também ter contribuído para as diferenças encontradas nos resultados.

Sob esse especto, uma vez que o processo de aquisição de habilidades motoras envolve um desenvolvimento hierárquico, o comportamento parcialmente desordenado é gradualmente organizado em torno de uma meta e de consequências ambientais, o que leva a uma maior consistência da habilidade aprendida (Doğan, 2009; Freudenheim et al., 2005; Yamashiro et al., 2013).

\section{CONCLUSÃO}

Análises em torno da aquisição de habilidades motoras em situações de meio-líquido, bem como das capacidades motoras subjacentes ao sucesso na habilidade executada, tanto com finalidades pedagógicas, quanto para investigações científicas, são essenciais para profissionais e pesquisadores interessados em testar e classificar os aprendizes em relação ao nível de aprendizagem. Os resultados do presente estudo permitem concluir que o tempo de reação é uma capacidade perceptivo-motora treinável, importante para o sucesso na performance do nado crawl, uma vez que foram encontradas diferenças estatísticas significativas quando comparados grupos de iniciantes e avançados, indicando que no estágio avançado os aprendizes possuem melhores tempos de resposta. Diante destes pressupostos ressalta-se a necessidade de professores e técnicos de natação observarem a importância do desenvolvimento das capacidades motoras, tanto quanto, da habilidade motora específica, com maior atenção as mudanças que ocorrem nos níveis de habilidade dos aprendizes durante o processo de aquisição e aprimoramento do nado.

Agradecimentos:

Nada a declarar.

\section{Conflito de Interesses:}

Nada a declarar.

\section{Financiamento:}

Nada a declarar 
18 | GR Smidt, ES Soares, AC Piovesan, AC Alpes, MR Silva, ST Corazza

\section{REFERÊNCIAS}

Araujo, R. R. de, \& Carreiro, L. R. R. (2009). Orientação voluntária e automática da atenção e indicadores de desatenção e hiperatividade em adultos. Avaliação Psicológica, 8(3), 325-336.

Bôscolo, E. F. M., Santos, L. M., \& Oliveira, S. L. de. (2011). Natação para adultos: a adaptação ao meio aquático fundamentada no aprendizado das habilidades motoras aquáticas básicas. $R e$ vista Educação - UnG, 6(1), 21-28.

Canfield, J. T. (2000). Aprendizagem de habilidades motoras II: o que muda com a prática? Revista Paulista de Educação Física, (S3), 72-78.

Corazza, S. T., Pereira, É. F., Villis, J. M. C., \& Katzer, J. I. (2006). Creating and validating a test for measuring motor performance of the crawl swimming style. Brazilian Journal of Kinanthropometry and Human Performance, 8(3), 73-78.

Dayan, E., \& Cohen, L. G. (2011). Neuroplasticity subserving motor skill learning. Neuron, 72(3), 443-454. http://doi.org/10.1016/j.neuron.2011.10.008

Deary, I. J., Liewald, D., \& Nissan, J. (2011). A free, easy-to-use, computer-based simple and fourchoice reaction time programme: the DearyLiewald reaction time task. Behavior Research Methods, 43(1), 258-268. http://doi.org/10.3758/s13428-010-0024-1

Doğan, B. (2009). Multiple-choice reaction and visual perception in female and male elite athletes. The Journal of Sports Medicine and Physical Fitness, 49(1), 91-96.

Droit-Volet, S., \& Zélanti, P. S. (2013). Development of time sensitivity and information processing speed. Plos One, 8(8), e71424. http://doi.org/10.1371/journal.pone.0071424

Fortes, L. de S., Miranda, V. P. N., Amaral, A. C. S., \& Ferreira, M. E. C. (2011). Body dissatisfaction of teen athletes and non-athletes. Jornal Brasileiro de Psiquiatria, 60(4), 309-314. http://doi.org/10.1590/S004720852011000400012

Freudenheim, A. M., Basso, L., Xavier-Filho, E., Silva, C., Madureira, F., \& Manoel, E. (2005). Organização temporal da braçada do nado crawl: iniciantes «versus» avançados. Revista Brasileira de Ciência e Movimento, 15(2), 75-84.

Hedvall, Å., Fernell, E., Holm, A., Åsberg Johnels, J., Gillberg, C., \& Billstedt, E. (2013). Autism, processing speed, and adaptive functioning in preschool children. TheScientificWorldJournal, 2013, 158263. http://doi.org/10.1155/2013/158263

Kaufman, S. B., DeYoung, C. G., Gray, J. R., Brown, J., \& Mackintosh, N. (2009). Associative learning predicts intelligence above and beyond working memory and processing speed. Intelligence, 37(4), 374-382. http://doi.org/10.1016/j.intell.2009.03.004

Lidor, R., Argov, E., \& Daniel, S. (1998). An exploratory study of perceptual-motor abilities of women: novice and skilled players of team handball. Perceptual and Motor Skills, 86(1), 279-288.

http://doi.org/10.2466/pms.1998.86.1.279

Macedo, E. C. de, Covre, P., Orsati, F. T., Oliveira, M. O. de, \& Schwartzman, J. S. (2007). Analysis of the eye movement patterns in visual search tasks: effect of familiarity and stimulus features. Arquivos Brasileiros de Oftalmologia, 70(1), 31-36. http://doi.org/10.1590/S000427492007000100006

Magill, R. A. (2000). Aprendizagem motora: conceitos e aplicações ( $5^{\mathrm{a}}$ ed.). São Paulo: Edgard Blücher.

Martins, A., Dascal, J., Bruzi, A., Caldeira, M., \& Turetta, C. (2010). Interferência de tarefas motoras com diferentes demandas de processamento sobre o tempo de reação de idosos e adultos jovens. Brazilian Journal of Sports and Exercise Research, 1(2), 127-134.

Miyamoto, R., \& Meira, C. (2004). Tempo de reação e tempo das provas de 50 e 100 metros rasos do atletismo em federados e não federados. Revista Portuguesa da Ciência do Desporto, 4(3), 4248.

Morales, A. P., Maciel, R. N., Silva, V. F. da, \& Silva, A. P. R. de S. e. (2011). Tempo de reação motora no voleibol. Biológicas \& Saúde, 1(3), 4249.

Mori, S., Ohtani, Y., \& Imanaka, K. (2002). Reaction times and anticipatory skills of karate athletes. Human Movement Science, 21(2), 213-230.

Pellegrini, A. M. (2000). A Aprendizagem de habilidades motoras I: o que muda com a prática? Rev. paul. educ. fís, S3, 29-34.

Penke, L., Muñoz Maniega, S., Murray, C., Gow, A. J., Hernández, M. C. V., Clayden, J. D., ... Deary, I. J. (2010). A general factor of brain white matter integrity predicts information processing speed in healthy older people. The Journal of Neuroscience: The Official Journal of the Society for Neuroscience, 30(22), 7569-7574. http://doi.org/10.1523/JNEUROSCI.155310.2010

Pereira, E. F., Dias, J., \& Corazza, S. T. (2007). Creation, development and analysis of reproductiveness of test to evaluate simple and choice reaction times. Fiep Bulletin, 77, 613-615.

Pereira, E. F., Teixeira, C. S., \& Corazza, S. T. (2012). A estrutura do movimento e a aprendizagem das habilidades motoras. Atividade Física Lazer \& Qualidade Vida: Revista de Educação Física, 2(2), 43-57.

Pereira, E. F., Teixeira, C., Villis, J., \& Corazza, S. T. (2009). Tempo de reação e desempenho motor do nado crawl em diferentes estágios de aprendizagem. Fisioterapia e Movimento, 22(4), 585594.

Schmidt, R. A., \& Wrisberg, C. A. (2001). Aprendizagem e performance motora: uma abordagem da aprendizagem baseada no problema ( $2^{\mathrm{a}} \mathrm{ed}$.). Porto Alegre: ArtMed. 
Seifert, L., Boulesteix, L., Chollet, D., \& Vilas-Boas, J. P. (2008). Differences in spatial-temporal parameters and arm-leg coordination in butterfly stroke as a function of race pace, skill and gender. Human Movement Science, 27(1), 96-111. http://doi.org/10.1016/j.humov.2007.08.001

Seifert, L., Leblanc, H., Chollet, D., \& Delignières, D. (2010). Inter-limb coordination in swimming: effect of speed and skill level. Human Movement Science, 29(1), 103-113. http://doi.org/10.1016/j.humov.2009.05.003

Takeuchi, H., Taki, Y., Hashizume, H., Sassa, Y., Nagase, T., Nouchi, R., \& Kawashima, R. (2011). Effects of training of processing speed on neural systems. The Journal of Neuroscience: The Official Journal of the Society for Neuroscience, 31(34), 12139-12148. http://doi.org/10.1523/JNEUROSCI.294811.2011

Thomas, J. R., Nelson, J. K., \& Silverman, S. J. (2007). Métodos de pesquisa em atividade física ( $5^{\mathrm{a}}$ ed.). Porto Alegre: ArtMed.
Vaghetti, C. A. O., Roesler, H., \& Andrade, A. (2007). Tempo de reação simples auditivo e visual em surfistas com diferentes níveis de habilidade: comparação entre atletas profissionais, amadores e praticantes. Revista Brasileira de Medicina do Esporte, 13(2), 81-85. http://doi.org/10.1590/S151786922007000200003

Wulf, G., Shea, C., \& Lewthwaite, R. (2010). Motor skill learning and performance: a review of influential factors. Medical Education, 44(1), 7584. http://doi.org/10.1111/j.13652923.2009.03421.x

Yamashiro, K., Sato, D., Onishi, H., Yoshida, T., Horiuchi, Y., Nakazawa, S., \& Maruyama, A. (2013). Skill-specific changes in somatosensory-evoked potentials and reaction times in baseball players. Experimental Brain Research, 225(2),

197-203. http://doi.org/10.1007/s00221-012-3361-8 